\title{
THE SUPERCRITICAL GENERALIZED KDV EQUATION: GLOBAL WELL-POSEDNESS IN THE ENERGY SPACE AND BELOW
}

\author{
Luiz G. Farah, Felipe Linares, and Ademir Pastor
}

\begin{abstract}
We consider the generalized Korteweg-de Vries (gKdV) equation $\partial_{t} u+$ $\partial_{x}^{3} u+\mu \partial_{x}\left(u^{k+1}\right)=0$, where $k \geq 5$ is an integer number and $\mu= \pm 1$. In the focusing case $(\mu=1)$, we show that if the initial data $u_{0}$ belongs to $H^{1}(\mathbb{R})$ and satisfies $E\left(u_{0}\right)^{s_{k}} M\left(u_{0}\right)^{1-s_{k}}<E(Q)^{s_{k}} M(Q)^{1-s_{k}}, E\left(u_{0}\right) \geq 0$, and $\left\|\partial_{x} u_{0}\right\|_{L^{2}}^{s_{k}}\left\|u_{0}\right\|_{L^{2}}^{1-s_{k}}<$ $\left\|\partial_{x} Q\right\|_{L^{2}}^{s_{k}}\|Q\|_{L^{2}}^{1-s_{k}}$, where $M(u)$ and $E(u)$ are the mass and energy, then the corresponding solution is global in $H^{1}(\mathbb{R})$. Here, $s_{k}=\frac{(k-4)}{2 k}$ and $Q$ is the ground state solution corresponding to the $\mathrm{gKdV}$ equation. In the defocusing case $(\mu=-1)$, if $k$ is even, we prove that the Cauchy problem is globally well-posed in the Sobolev spaces $H^{s}(\mathbb{R})$, $s>\frac{4(k-1)}{5 k}$.
\end{abstract}

\section{Introduction}

Consider the Initial Value Problem (IVP) associated with the supercritical generalized Korteweg-de Vries (gKdV) equation, i.e.,

$$
\left\{\begin{array}{l}
\partial_{t} u+\partial_{x}^{3} u+\mu \partial_{x}\left(u^{k+1}\right)=0, \quad x \in \mathbb{R}, t>0 \\
u(x, 0)=u_{0}(x)
\end{array}\right.
$$

where $\mu= \pm 1$.

Local well-posedness of the Cauchy problem (1.1) (with $k \geq 1$ ) has been studied by many authors in recent years. We refer the reader to Kenig, Ponce, and Vega [17], [18] for a complete set of sharp results.

Our main interest here is on global well-posedness. Let us briefly recall the best results available in the literature. For $k=1$ and $k=2$, global well-posedness was established by Colliander, Keel, Staffilani, Takaoka, and Tao [6] for data, respectively, in $H^{s}(\mathbb{R}), s>-3 / 4$ and $H^{s}(\mathbb{R}), s>1 / 4$, and by Guo [12] for data, respectively, in $H^{-3 / 4}(\mathbb{R})$ and $H^{1 / 4}(\mathbb{R})$. These results show to be sharp in view of the work of Kenig, Ponce, and Vega [19] (see also [1], [4], [28]).

The case $k=3$ was dealt with by Grünrock, Panthee, and Silva [10], where the authors showed global well-posedness in $H^{s}(\mathbb{R}), s>-1 / 42$. It should be pointed out that for $k=3$, Tao [27] established a local existence result in $\dot{H}^{-\frac{1}{6}}(\mathbb{R})$, the critical (scale-invariant) space, therefore for small data the solutions extend globally. For recent progress in this case we refer to Koch and Marzuola [21]. Under "sharp

Received by the editors September 14, 2010.

The first author was partially supported by CNPq and FAPEMIG/Brazil.

The second author was partially supported by CNPq/Brazil.

The third author was partially supported by $\mathrm{CNPq} /$ Brazil. 
smallness condition", the critical case $k=4$ was studied by Fonseca, Linares, and Ponce in [9]. There it was established global well-posedness in $H^{s}(\mathbb{R}), s>3 / 4$. Farah [8] used the I-method of [6], to further lower the regularity of the initial data to $s>3 / 5$. Recently, Miao, Shao, $\mathrm{Wu}$, and Xu [25], improved the latter result to initial data in $H^{s}(\mathbb{R}), s>6 / 13$. Their method of proof combines the I-method with a multilinear correction analysis. For $k=4$, Kenig, Ponce, and Vega [17] showed local well-posedness for data in $L^{2}$ (the critical space in this case), which for small data yield global solutions. Finally, we should mention that for $k=4$, Merle [24] and Martel and Merle [23] proved the existence of real-valued solutions of (1.1) in $H^{1}(\mathbb{R})$ corresponding to data in $u_{0} \in H^{1}(\mathbb{R})$ with $\left\|u_{0}\right\|_{L^{2}}>\|Q\|_{L^{2}}$ that blow-up. For $k>4$ it is an outstanding open problem.

As far as we are concerned, for $k \geq 5$, no global results below the energy space are available. Not even a precise description of the conditions to obtain $H^{1}$ global solutions. These facts motivate the present study.

To start with the local results, using a scaling argument let us motivate what should be the Sobolev spaces to studying (1.1). Note if $u$ is a solution of (1.1), then, for any $\lambda>0, u_{\lambda}(x, t)=\lambda^{2 / k} u\left(\lambda x, \lambda^{3} t\right)$ is also a solution with initial data $u_{\lambda}(x, 0)=\lambda^{2 / k} u_{0}(\lambda x)$. Moreover,

$$
\left\|u_{\lambda}(\cdot, 0)\right\|_{\dot{H}^{s}}=\lambda^{s+2 / k-1 / 2}\left\|u_{0}\right\|_{\dot{H}^{s}} .
$$

Thus, for each $k$ fixed, the scale-invariant Sobolev space is $\dot{H}^{s_{k}}, s_{k}=(k-4) / 2 k$. Therefore, the natural Sobolev spaces to studying (1.1) are $H^{s}, s>s_{k}=(k-4) / 2 k$. Actually, this question has already been addressed by Kenig, Ponce, and Vega [17]. More precisely, they show the following.

Theorem 1.1. Let $k>4$ and $s>s_{k}=(k-4) / 2 k$. Then for any $u_{0} \in H^{s}(\mathbb{R})$ there exist $T=T\left(\left\|u_{0}\right\|_{H^{s}}\right)>0$ (with $T(\rho ; s) \rightarrow \infty$ as $\rho \rightarrow 0$ ) and a unique strong solution $u(\cdot)$ of the IVP (1.1) satisfying:

$$
\begin{gathered}
u \in C\left([-T, T]: H^{s}(\mathbb{R})\right), \\
\|u\|_{L_{x}^{5} L_{T}^{10}}+\left\|D_{x}^{s} u\right\|_{L_{x}^{5} L_{T}^{10}}<\infty \\
\left\|u_{x}\right\|_{L_{x}^{\infty} L_{T}^{2}}+\left\|D_{x}^{s} u_{x}\right\|_{L_{x}^{\infty} L_{T}^{2}}<\infty,
\end{gathered}
$$

and

$$
\left\|D_{t}^{\gamma_{k}} D_{x}^{\alpha_{k}} D_{t}^{\beta_{k}} u\right\|_{L_{x}^{p_{k}} L_{T}^{q_{k}}}<\infty
$$

where

$$
\begin{gathered}
\alpha_{k}=\frac{1}{10}-\frac{2}{5 k}, \quad \beta_{k}=\frac{3}{10}-\frac{6}{5 k}, \quad \gamma_{k}=\gamma_{k}(s)=\frac{s-s_{k}}{3} \\
\frac{1}{p_{k}}=\frac{2}{5 k}+\frac{1}{10}, \quad \frac{1}{q_{k}}=\frac{3}{10}-\frac{4}{5 k} .
\end{gathered}
$$

Furthermore, given $T^{\prime} \in(0, T)$ there exists a neighborhood $V$ of $u_{0}$ in $H^{s}(\mathbb{R})$ such that the map $u_{0} \mapsto \tilde{u}(t)$ from $V$ into the class defined by (1.2)-(1.4) with $T^{\prime}$ instead of $T$ is smooth. 
The method to prove Theorem 1.1 combines smoothing effects and Strichartz-type estimates together with the Banach contraction principle. As a matter of fact, the original theorem stated in [17] differs slightly in the function spaces. Here, we will give a skech of the proof of Theorem 1.1 in this function spaces setting.

Remark 1.2. It should be observed that in [17] the authors also showed a local result for initial data in $\dot{H}^{s_{k}}(\mathbb{R}), s_{k}$ as above, but $T=T\left(u_{0}\right)$, that is, the existence time $T$ depends on $u_{0}$ itself and not on $\left\|u_{0}\right\|_{\dot{H}^{s_{k}}}$ (see also [1]) and that this is global for small real or complex-valued data.

Once Theorem 1.1 is established, a natural question presents itself: can the real solutions be extended globally-in-time? Such a question has mathematical and physical interest and it has been widely studied in the past few years.

By observing that the flow of the gKdV equation is conserved by the quantities

$$
\text { Mass } \equiv M(u(t))=\int u^{2}(t) d x
$$

and

$$
\text { Energy } \equiv E(u(t))=\frac{1}{2} \int\left(\partial_{x} u\right)^{2}(t) d x-\frac{\mu}{k+2} \int u^{k+2}(t) d x,
$$

one can partially answer this question for solutions in $H^{1}(\mathbb{R})$ if the initial data is small (see [17, Theorem 2.15]). Note that the case where $\mu=-1$ and $k$ even is, in some sense, special. Indeed, we have the following result.

Corollary 1.3. Let $k>4$ and $s>s_{k}=(k-4) / 2 k$. If $\mu=-1$ and $k$ is even, for $u_{0} \in H^{1}$ of arbitrary size, there exists a unique strong solution $u(\cdot)$ of the IVP (1.1) satisfying

$$
u \in C\left(\mathbb{R}: H^{1}(\mathbb{R})\right) \cap L^{\infty}\left(\mathbb{R}: H^{1}(\mathbb{R})\right) .
$$

We have two main goals in this paper. The first one is to make precise the $H^{1}$-size of the initial data to construct global $H^{1}$ solutions when $\mu=1$ or $\mu=-1$ and $k$ odd. The second one is to loosen the regularity requirements on the initial data which ensure global-in-time solutions for the IVP (1.1) when $\mu=-1$ and $k$ even. Below we also explain why we cannot apply the same method when $\mu=1$ or $\mu=-1$ and $k$ odd (see Remark 5.9).

We consider first the focusing case $\mu=1$ or the defocusing case $\mu=-1$ with $k$ odd. In these cases, it is not clear how large is the size of the initial data in $H^{1}$ to obtain global solutions. The next theorem shows us how small the initial data should be.

Theorem 1.4. Let $u_{0} \in H^{1}(\mathbb{R})$. Let $k>4$ and $s_{k}=(k-4) / 2 k$. Suppose that

$$
E\left(u_{0}\right)^{s_{k}} M\left(u_{0}\right)^{1-s_{k}}<E(Q)^{s_{k}} M(Q)^{1-s_{k}}, \quad E\left(u_{0}\right) \geq 0 .
$$

If

$$
\left\|\partial_{x} u_{0}\right\|_{L^{2}}^{s_{k}}\left\|u_{0}\right\|_{L^{2}}^{1-s_{k}}<\left\|\partial_{x} Q\right\|_{L^{2}}^{s_{k}}\|Q\|_{L^{2}}^{1-s_{k}},
$$

then for any $t$ as long as the solution exists,

$$
\left\|\partial_{x} u(t)\right\|_{L^{2}}^{s_{k}}\left\|u_{0}\right\|_{L^{2}}^{1-s_{k}}=\left\|\partial_{x} u(t)\right\|_{L^{2}}^{s_{k}}\|u(t)\|_{L^{2}}^{1-s_{k}}<\left\|\partial_{x} Q\right\|_{L^{2}}^{s_{k}}\|Q\|_{L^{2}}^{1-s_{k}},
$$


where $Q$ is unique positive even solution of the elliptic equation

$$
\Delta Q-Q+Q^{k+1}=0 .
$$

This in turn implies that $H^{1}$ solutions exist globally in time.

To prove Theorem 1.4, we follow closely the arguments in Holmer and Roudenko [13], which were inspired by those introduced by Kenig and Merle [15].

Next we consider the defocusing case $\mu=-1$ with $k$ even. Our main result is the following.

Theorem 1.5. Let $\mu=-1$ and assume that $k$ is even. Let $u_{0} \in H^{s}(\mathbb{R}), s>\frac{4(k-1)}{5 k}$. Then, the local solution in Theorem 1.1 can be extended to any time interval. Moreover, for all $T>0$, the solution satisfies

$$
\sup _{t \in[0, T]}\left\{\|u(t)\|_{H^{s}}^{2}\right\} \leq C(1+T)^{\frac{(1+4 / k)(1-s)}{5 s-4(k-1) / k}+},
$$

where the constant $C$ depends only on $s$ and $\left\|u_{0}\right\|_{H^{s}}$.

Remark 1.6. Note that when $k=4$ we recover the result proved in Farah [8].

Here we use the approach introduced by Colliander, Keel, Staffilani, Takaoka and Tao in [5], the so-called $I$-method. We also explain why the refined approach introduced by the same authors in [6] cannot be used to improve our global result stated in Theorem 1.5 (see Remark 5.2).

Note that when $u_{0} \in H^{s}(\mathbb{R})$ with $s<1$ in (1.1), the energy (1.9) could be infinite, and so the conservation law (1.9) is meaningless. To overcome this difficulty, by following the $I$-method scheme, we introduce a modified energy functional which is also defined for less regular functions. Unfortunately, this new functional is not strictly conserved, but we can show that it is almost conserved in time. When one is able to control its growth in time explicitly, this allows to iterate a modified local existence theorem to continue the solution to any time $T$.

The plan of this paper is as follows. In the next section we introduce some notation and preliminaries. In Section 3 we prove Theorem 1.1. Next, in Section 4, we show Theorem 1.4. The result of global well-posedness in Theorem 1.5 is proved in Section 5.

\section{Notation and Preliminaries}

Let us start this section by introducing the notation used throughout the paper. We use $c$ to denote various constants that may vary line by line. Given any positive numbers $a$ and $b$, the notation $a \lesssim b$ means that there exists a positive constant $c$ such that $a \leq c b$. Also, we denote $a \sim b$ when, $a \lesssim b$ and $b \lesssim a$. We use $a+$ and $a-$ to denote $a+\varepsilon$ and $a-\varepsilon$, respectively, for arbitrarily small $\varepsilon>0$.

We use $\|\cdot\|_{L^{p}}$ to denote the $L^{p}(\mathbb{R})$ norm. If necessary, we use subscript to inform which variable we are concerned with. The mixed norm $L_{t}^{q} L_{x}^{r}$ of $f=f(x, t)$ is defined as

$$
\|f\|_{L_{t}^{q} L_{x}^{r}}=\left(\int\|f(\cdot, t)\|_{L_{x}^{r}}^{q} d t\right)^{1 / q}
$$

with the usual modifications when $q=\infty$ or $r=\infty$. The $L_{x}^{r} L_{t}^{q}$ norm is similarly defined. 
We define the spatial Fourier transform of $f(x)$ by

$$
\hat{f}(\xi)=\int_{\mathbb{R}} e^{-i x \xi} f(x) d x
$$

and the space-time Fourier transform of $u(x, t)$ by

$$
\widetilde{u}(\xi, \tau)=\int_{\mathbb{R}} \int_{\mathbb{R}} e^{-i(x \xi+t \tau)} u(x, t) d t d x .
$$

Note that the derivative $\partial_{x}$ is conjugated to multiplication by $i \xi$ by the Fourier transform.

The set of Schwartz functions is represented by $\mathcal{S}(\mathbb{R})$. We shall also define $D^{s}$ and $J^{s}$ to be, respectively, the Fourier multipliers with symbols $|\xi|^{s}$ and $\langle\xi\rangle^{s}=(1+|\xi|)^{s}$. Thus, the norm in the Sobolev space $H^{s}(\mathbb{R})$ is given by

$$
\|f\|_{H^{s}} \equiv\left\|J^{s} f\right\|_{L_{x}^{2}}=\left\|\langle\xi\rangle^{s} \hat{f}\right\|_{L_{\xi}^{2}}
$$

We also define the spaces $X_{s, b}(\mathbb{R} \times \mathbb{R})$ on $\mathbb{R} \times \mathbb{R}$ through the norm

$$
\|F\|_{X_{s, b}(\mathbb{R} \times \mathbb{R})}=\left\|\left\langle\tau-\xi^{3}\right\rangle^{b}\langle\xi\rangle^{s} \widetilde{F}\right\|_{L_{\xi, \tau}^{2}} .
$$

These spaces were introduced in the study of nonlinear dispersive wave problems by Bourgain [2].

For any interval $I$, we define the localized $X_{s, b}(I \times \mathbb{R})$ spaces by

$$
\|u\|_{X_{s, b}(I \times \mathbb{R})}=\inf \left\{\|w\|_{X_{s, b}(\mathbb{R} \times \mathbb{R})}: w(t)=u(t) \text { on } I\right\} .
$$

We often abbreviate $\|u\|_{X_{s, b}}$ and $\|u\|_{X_{s, b}^{I}}$, respectively, for $\|u\|_{X_{s, b}(\mathbb{R} \times \mathbb{R})}$ and $\|u\|_{X_{s, b}(I \times \mathbb{R})}$.

Let us introduce now some useful lemmas and inequalities. In what follows, $U(t)$ denotes the group associated with the linear $\mathrm{KdV}$ equation, that is, for any $u_{0}, U(t) u_{0}$ is the solution of the linear problem

$$
\left\{\begin{array}{l}
\partial_{t} u+\partial_{x}^{3} u=0, \quad x \in \mathbb{R}, t \in \mathbb{R}, \\
u(x, 0)=u_{0}(x) .
\end{array}\right.
$$

We begin by recalling the results necessary to prove Theorem 1.1 and some linear estimates in Bourgain's spaces which will be needed later.

Lemma 2.1. Let $p, q$, and $\alpha$ be such that

$$
\frac{1}{p}+\frac{1}{2 q}=\frac{1}{4}, \quad \alpha=\frac{2}{q}-\frac{1}{p}, \quad 1 \leq p, q \leq \infty, \quad-\frac{1}{4} \leq \alpha \leq 1 .
$$

Then

$$
\left\|D_{x}^{\alpha} U(t) u_{0}\right\|_{L_{x}^{p} L_{t}^{q}} \lesssim\left\|u_{0}\right\|_{L_{x}^{2}}
$$

In particular, for $(p, q, \alpha)=(2, \infty, 1)$, the dual version of $(2.16)$ reads as follows:

$$
\left\|\partial_{x} \int_{0}^{t} U\left(t-t^{\prime}\right) g\left(\cdot, t^{\prime}\right) d t^{\prime}\right\|_{L_{x}^{2}} \lesssim\|g\|_{L_{x}^{1} L_{t}^{2}}
$$

Proof. See [20, Proposition 2.1]. 
Lemma 2.2. Let $p_{i}, q_{i}, \alpha_{i}, i=1,2$, satisfy the relations in (2.15). Then

$$
\left\|D_{x}^{\alpha_{1}} \int_{0}^{t} U\left(t-t^{\prime}\right) g\left(\cdot, t^{\prime}\right) d t^{\prime}\right\|_{L_{x}^{p_{1}} L_{t}^{q_{1}}} \lesssim\left\|D_{x}^{-\alpha_{2}} g\right\|_{L_{x}^{p_{2}^{\prime}} L_{t}^{q_{2}^{\prime}}},
$$

where $p_{2}^{\prime}$ and $q_{2}^{\prime}$ are the Hölder conjugate of $p_{2}$ and $q_{2}$ respectively.

Proof. See [20, Proposition 2.2].

Lemma 2.3. If $g \in \mathcal{S}\left(\mathbb{R}^{2}\right)$, then

$$
\|g\|_{L_{x}^{5 k / 4} L_{t}^{5 k / 2}} \lesssim\left\|D_{x}^{\alpha_{k}} D_{t}^{\beta_{k}} g\right\|_{L_{x}^{p_{k} L_{t}^{q_{k}}}},
$$

where $\alpha_{k}, \beta_{k}, p_{k}$, and $q_{k}$ are defined as in (1.6)-(1.7).

Proof. See [17, Lemma 3.15].

Lemma 2.4. Let $s_{k}, \alpha_{k}, \beta_{k}, p_{k}$, and $q_{k}$ be as in Theorem 1.1. Let $\alpha \geq 0, \beta \geq 0$, and $u_{0} \in \mathcal{S}(\mathbb{R})$. Then

$$
\left\|D_{x}^{\alpha} D_{t}^{\beta / 3} D_{x}^{\alpha_{k}} D_{t}^{\beta_{k}} U(t) u_{0}\right\|_{L_{x}^{p_{k}} L_{t}^{q_{k}}} \lesssim\left\|D_{x}^{\alpha+\beta} D_{x}^{s_{k}} u_{0}\right\|_{L_{x}^{2}} .
$$

Proof. See [17, Lemmas 3.14 and 3.16].

Lemma 2.5. Let $s \geq s_{k}$. Let $p_{k}$ and $q_{k}$ be as in Theorem 1.1. The following estimate is fulfilled

$$
\begin{aligned}
& \left\|D_{x}^{s}\left(u^{k} \partial_{x} u\right)\right\|_{L_{x}^{5 / 4} L_{t}^{10 / 9}} \lesssim\left\|D_{x}^{\alpha_{k}} D_{t}^{\beta_{k}} u\right\|_{L_{x}^{p_{k}} L_{t}^{q_{k}}}^{k}\left\|D_{x}^{s} \partial_{x} u\right\|_{L_{x}^{\infty} L_{t}^{2}} \\
& +\left\|D_{x}^{\alpha_{k}} D_{t}^{\beta_{k}} u\right\|_{L_{x}^{p_{k}} L_{t}^{q_{k}}}^{k-1}\|u\|_{L_{x}^{5 k / 4} L_{t}^{5 k / 2}}\left\|D_{x}^{s} \partial_{x} u\right\|_{L_{x}^{\infty} L_{t}^{2}} .
\end{aligned}
$$

Proof. See proof of Proposition 6.1 in [17].

Lemma 2.6. Let $0<\alpha<1$ and $p, p_{1}, p_{2}, q, q_{1}, q_{2} \in(1, \infty)$ with $\frac{1}{p}=\frac{1}{p_{1}}+\frac{1}{p_{2}}$ and $\frac{1}{q}=\frac{1}{q_{1}}+\frac{1}{q_{2}}$. Then,

(i)

$$
\left\|D_{x}^{\alpha}(f g)-f D_{x}^{\alpha} g-g D_{x}^{\alpha} f\right\|_{L_{x}^{p} L_{T}^{q}} \lesssim\left\|D_{x}^{\alpha} f\right\|_{L_{x}^{p_{1}} L_{T}^{q_{1}}}\|g\|_{L_{x}^{p_{2}} L_{T}^{q_{2}}} .
$$

The same still holds if $p=1$ and $q=2$.

(ii)

$$
\left\|D_{x}^{\alpha} F(f)\right\|_{L_{x}^{p} L_{T}^{q}} \lesssim\left\|D_{x}^{\alpha} f\right\|_{L_{x}^{p_{1}} L_{T}^{q_{1}}}\left\|F^{\prime}(f)\right\|_{L_{x}^{p_{2}} L_{T}^{q_{2}}}
$$

Proof. See [17, Theorems A.6, A.8, and A.13].

Next, we introduce some inequalities to prove a variant of Theorem 1.1. These tools will be used in Section 5.

Lemma 2.7 (Strichartz estimates). The following inequalities hold.

(i)

$$
\|u\|_{X_{0,-\frac{1}{2}++}} \lesssim\|u\|_{L_{x}^{5 / 4+} L_{t}^{10 / 9+},}
$$

where $a++=a+2 \varepsilon$ for sufficiently small $\varepsilon>0$. 
(ii)

$$
\begin{aligned}
&\|u\|_{L_{x, t}^{p}} \lesssim\|u\|_{X_{\alpha(p), \frac{1}{2}+},} \\
& \text { where } p>8 \text { and } \alpha(p)=\left(\frac{1}{2}+\right)\left(\frac{p-8}{p}\right) .
\end{aligned}
$$

Remark 2.8. Note that the numbers $\varepsilon>0$ that appear implicitly in the fractions $1 / 2++, 5 / 4+$ or $10 / 9+$ can be chosen arbitrarily.

Proof. Recall the Strichartz estimates (see [16])

$$
\|u\|_{L_{x, t}^{8}} \lesssim\|u\|_{X_{0, \frac{1}{2}+}}
$$

and

$$
\|u\|_{L_{x}^{5} L_{t}^{10}} \lesssim\|u\|_{X_{0, \frac{1}{2}+}}
$$

By duality

$$
\|u\|_{X_{0,-\frac{1}{2}-}} \lesssim\|u\|_{L_{x}^{5 / 4} L_{t}^{10 / 9}}
$$

which interpolated with the trivial estimate

$$
\|u\|_{X_{0,0}} \lesssim\|u\|_{L_{x, t}^{2}}
$$

yields (2.17)

Interpolation between (2.19) and $\|u\|_{L_{x, t}^{\infty}} \lesssim\|u\|_{X_{\frac{1}{2}+\frac{1}{2}+}}$ gives us (2.18).

Lemma 2.9. The following inequalities hold.

(i)

$$
\left\|D_{x}^{\alpha_{k}-} D_{t}^{\beta_{k}-} u\right\|_{L_{x}^{p_{k}+} L_{t}^{q_{k}+}} \lesssim\|u\|_{X_{s_{k}+, \frac{1}{2}+}}
$$

and

$$
\|u\|_{L_{x}^{5 k / 4+} L_{t}^{5 k / 2+}} \lesssim\left\|D_{x}^{\alpha_{k}-} D_{t}^{\beta_{k}-} u\right\|_{L_{x}^{p_{k}+} L_{t}^{q_{k}+}} .
$$

and

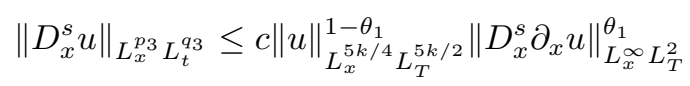

and

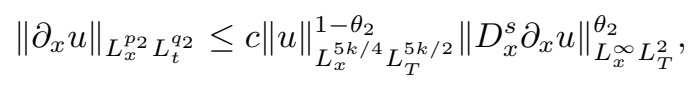

where

$$
\frac{1}{p_{2}}+\frac{1}{p_{3}}=\frac{4}{5 k}, \quad \frac{1}{q_{2}}+\frac{1}{q_{3}}=\frac{1}{2}+\frac{2}{5 k}
$$

and $\theta_{1}=\frac{s}{1+s}, \theta_{2}=\frac{1}{1+s}$, both $\theta_{1}$ and $\theta_{2}$ are in $(0,1)$ with $\theta_{1}+\theta_{2}=1$. 
Proof. Recall that from Lemmas 2.3 and 2.4,

$$
\left\|D_{x}^{\alpha_{k}} D_{t}^{\beta_{k}} u\right\|_{L_{x}^{p_{k} L_{t}^{q_{k}}}} \lesssim\|u\|_{X_{s_{k}, \frac{1}{2}+}}
$$

and

$$
\|u\|_{L_{x}^{5 k / 4} L_{t}^{5 k / 2}} \lesssim\left\|D_{x}^{\alpha_{k}} D_{t}^{\beta_{k}} u\right\|_{L_{x}^{p_{k}} L_{t}^{q_{k}}} .
$$

Interpolating, respectively, with $\|u\|_{L_{x, t}^{\infty}} \lesssim\|u\|_{X_{\frac{1}{2}+\frac{1}{2}+}}$ and $\|u\|_{L_{x, t}^{\infty}} \lesssim\|u\|_{L_{x, t}^{\infty}}$ we obtain (2.20) and (2.21). Moreover, an interpolation yield (2.22) and (2.23).

Next we recall some linear estimates in Bourgain's spaces which will be needed later. Let $\varphi$ be a cutoff function satisfying $\varphi \in C_{0}^{\infty}(\mathbb{R}), 0 \leq \varphi \leq 1, \varphi \equiv 1$ in $[-1,1]$, $\operatorname{supp}(\varphi) \subseteq[-2,2]$. For $0<T<1$ define $\varphi_{T}(t)=\varphi(t / T)$.

Lemma 2.10 (Linear estimates). Let $-\frac{1}{2}<b^{\prime} \leq 0 \leq b \leq b^{\prime}+1$ and $0<T \leq 1$ then

(i) $\left\|\varphi(t) U(t) u_{0}\right\|_{X_{s, b}} \leq\left\|u_{0}\right\|_{H^{s}}$;

(ii) $\left\|\varphi_{T}(t) \int_{0}^{t} U\left(t-t^{\prime}\right) g\left(\cdot, t^{\prime}\right) d t^{\prime}\right\|_{X_{s, b}} \leq T^{1-\left(b-b^{\prime}\right)}\|g\|_{X_{s, b^{\prime}}}$.

Proof. See [2] and [18].

Finally, we have the following refined Strichartz estimate in the case of differing frequencies.

Lemma 2.11. Let $\psi_{1}, \psi_{2} \in X_{0, \frac{1}{2}+}$ be supported on spatial frequencies $\left|\xi_{i}\right| \sim N_{i}$, $i=1,2$. If $\max \left\{\left|\xi_{1}\right|,\left|\xi_{2}\right|\right\} \lesssim \min \left\{\left|\xi_{1}-\xi_{2}\right|,\left|\xi_{1}+\xi_{2}\right|\right\}$ for all $\xi_{i} \in \operatorname{supp}\left(\widehat{\psi}_{i}\right), i=1,2$, then

$$
\left\|\psi_{1} D_{x} \psi_{2}\right\|_{L_{x, t}^{2}} \lesssim\left\|\psi_{1}\right\|_{X_{0, \frac{1}{2}+}}\left\|\psi_{2}\right\|_{X_{0, \frac{1}{2}+}}
$$

Proof. See [8, Lemma 2.1] (see also Bourgain [3] and Grünrock [11]).

We now give some useful notation for multilinear expressions. If $n \geq 2$ is an even integer, we define a (spatial) $n$-multiplier to be any function $M_{n}\left(\xi_{1}, \ldots, \xi_{n}\right)$ on the hyperplane

$$
\Gamma_{n} \equiv\left\{\left(\xi_{1}, \ldots, \xi_{n}\right) \in \mathbb{R}^{n}: \xi_{1}+\cdots+\xi_{n}=0\right\},
$$

which we endow with the standard measure $\delta\left(\xi_{1}+\cdots+\xi_{n}\right)$, where $\delta$ is the Dirac delta.

If $M_{n}$ is an $n$-multiplier and $f_{1}, \ldots, f_{n}$ are functions on $\mathbb{R}$, we define the $n$-linear functional $\Lambda_{n}\left(M_{n} ; f_{1}, \ldots, f_{n}\right)$ by

$$
\Lambda_{n}\left(M_{n} ; f_{1}, \ldots, f_{n}\right)=\int_{\Gamma_{n}} M_{n}\left(\xi_{1}, \ldots, \xi_{n}\right) \prod_{j=1}^{n} \widehat{f}_{j}\left(\xi_{j}\right) .
$$

We will often apply $\Lambda_{n}$ to $n$ copies of the same function $u$ in which case the dependence upon $u$ may be suppressed in the notation: $\Lambda_{n}\left(M_{n} ; u, \ldots, u\right)$ may simply be written as $\Lambda_{n}\left(M_{n}\right)$.

If $M_{n}$ is symmetric, so does the $n$-linear functional $\Lambda_{n}\left(M_{n}\right)$. 
As an example, suppose that $u$ is an $\mathbb{R}$-valued function. By Plancherel's theorem, we can rewrite the energy (1.9) in terms of $n$-linear functionals as

$$
E(u(t))=-\frac{1}{2} \Lambda_{2}\left(\xi_{1} \xi_{2}\right)-\frac{\mu}{k+2} \Lambda_{k+2}(1) .
$$

The time derivative of a symmetric $n$-linear functional can be calculated explicitly if we assume that the function $u$ satisfies a particular PDE. The following statement may be directly verified by using the generalized KdV equation (1.1).

Proposition 2.12. Suppose u satisfies the generalized KdV equation (1.1) and that $M_{n}$ is a symmetric n-multiplier. Then $(2.26)$

$\frac{d}{d t} \Lambda_{n}\left(M_{n}\right)=\Lambda_{n}\left(M_{n} \alpha_{n}\right)-i n \mu \Lambda_{n+k}\left(M_{n}\left(\xi_{1}, \ldots, \xi_{n-1}, \xi_{n}+\cdots+\xi_{n+k}\right)\left(\xi_{n}+\cdots+\xi_{n+k}\right)\right)$, where $\alpha_{n} \equiv i\left(\xi_{1}^{3}+\cdots+\xi_{n}^{3}\right)$.

\section{Local well-posedness}

Our aim in this section is to establish Theorem 1.1. We use the contraction mapping principle. Define the metric spaces

$$
X_{T}=\left\{u \in C\left([0, T] ; H^{s}(\mathbb{R})\right):\|u\|_{s}<\infty\right\}
$$

and

$$
X_{T}^{a}=\left\{u \in X_{T}:\|u\|_{s} \leq a\right\},
$$

where

$$
\begin{aligned}
\|u\|_{s}= & \|u\|_{L_{T}^{\infty} H_{x}^{s}}+\|u\|_{L_{x}^{5} L_{T}^{10}}+\left\|D_{x}^{s} u\right\|_{L_{x}^{5} L_{T}^{10}} \\
& +\left\|\partial_{x} u\right\|_{L_{x}^{\infty} L_{T}^{2}}+\left\|D_{x}^{s} \partial_{x} u\right\|_{L_{x}^{\infty} L_{T}^{2}}+\left\|D_{t}^{\gamma_{k}} D_{x}^{\alpha_{k}} D_{t}^{\beta_{k}} u\right\|_{L_{x}^{p_{k}} L_{T}^{q_{k}}} .
\end{aligned}
$$

The parameters $T$ and $a$ will be appropriately chosen later. On $X_{T}$ consider the integral operator

$$
\Phi(u)(t):=U(t) u_{0}-\mu \int_{0}^{t} U\left(t-t^{\prime}\right) \partial_{x}\left(u^{k+1}\right)\left(t^{\prime}\right) d t^{\prime} .
$$

We only give the details to estimate the $\|\cdot\|_{L_{T}^{\infty} H^{s}}$-norm. From group properties and Lemma 2.1,

$$
\begin{aligned}
\|\Phi(u)\|_{L_{x}^{2}} & \leq\left\|u_{0}\right\|_{L_{x}^{2}}+\left\|\partial_{x} \int_{0}^{t} U\left(t-t^{\prime}\right) u^{k+1}\left(t^{\prime}\right) d t^{\prime}\right\|_{L_{x}^{2}} \\
& \lesssim \mid u_{0}\left\|_{L_{x}^{2}}+\right\| u^{k+1} \|_{L_{x}^{1} L_{T}^{2}} \\
& \lesssim\left\|u_{0}\right\|_{L_{x}^{2}}+\|u\|_{L_{x}^{5 k / 4} L_{T}^{5 k / 2}}^{k}\|u\|_{L_{x}^{5} L_{T}^{10}} .
\end{aligned}
$$

Now, from Lemma 2.3 and Sobolev's inequality it follows that

$$
\begin{aligned}
\|\Phi(u)\|_{L_{x}^{2}} & \leq\left\|u_{0}\right\|_{L_{x}^{2}}+\left\|D_{x}^{\alpha_{k}} D_{t}^{\beta_{k}} u\right\|_{L_{x}^{p_{k}} L_{T}^{q_{k}}}^{k}\|u\|_{L_{x}^{5} L_{T}^{10}} \\
& \lesssim\left\|u_{0}\right\|_{L_{x}^{2}}+T^{k \gamma_{k}}\left\|D_{t}^{\gamma_{k}} D_{x}^{\alpha_{k}} D_{t}^{\beta_{k}} u\right\|_{L_{x}^{p_{k}} L_{T}^{q_{k}}}^{k}\|u\|_{L_{x}^{5} L_{T}^{10}} \\
& \leq\left\|u_{0}\right\|_{L_{x}^{2}}+T^{k \gamma_{k}}\|u\|_{s}^{k+1} .
\end{aligned}
$$


Next, we estimate the $\dot{H}^{s}$-norm. Group properties and applications of Lemma 2.1 yield

$$
\begin{aligned}
\left\|D_{x}^{s} \Phi(u)\right\|_{L_{x}^{2}} & \leq\left\|D_{x}^{s} u_{0}\right\|_{L_{x}^{2}}+\left\|\partial_{x} \int_{0}^{t} U\left(t-t^{\prime}\right) D_{x}^{s}\left(u^{k+1}\right) d t^{\prime}\right\|_{L_{x}^{2}} \\
& \lesssim\left\|u_{0}\right\|_{L_{x}^{2}}+\left\|D_{x}^{s}\left(u^{k+1}\right)\right\|_{L_{x}^{1} L_{T}^{2}} .
\end{aligned}
$$

By applying Lemma 2.6 and then Lemma 2.3, we deduce

$$
\begin{aligned}
\left\|D_{x}^{s}\left(u^{k+1}\right)\right\|_{L_{x}^{1} L_{T}^{2}} \lesssim\left\|u^{k}\right\|_{L_{x}^{5 / 4} L_{T}^{5 / 2}}\left\|D_{x}^{s} u\right\|_{L_{x}^{5} L_{T}^{10}}+\left\|u D_{x}^{s}\left(u^{k}\right)\right\|_{L_{x}^{1} L_{T}^{2}} \\
\lesssim\|u\|_{L_{x}^{5 k / 4} L_{T}^{5 k / 2}}^{k}\left\|D_{x}^{s} u\right\|_{L_{x}^{5} L_{T}^{10}}+\|u\|_{L_{x}^{5 k / 4} L_{T}^{5 k / 2}}\left\|D_{x}^{s}\left(u^{k}\right)\right\|_{L_{x}^{p_{0}} L_{T}^{q_{0}}} \\
\lesssim\left\|D_{x}^{\alpha_{k}} D_{t}^{\beta_{k}} u\right\|_{L_{x}^{p_{k}} L_{T}^{q_{k}}}^{k}\left\|D_{x}^{s} u\right\|_{L_{x}^{5} L_{T}^{10}} \\
\quad+\left\|D_{x}^{\alpha_{k}} D_{t}^{\beta_{k}} u\right\|_{L_{x}^{p_{k}} L_{T}^{q_{k}}}\left\|D_{x}^{s} u\right\|_{L_{x}^{5} L_{T}^{10}}\left\|u^{k-1}\right\|_{L_{x}^{p_{1}} L_{T}^{q_{1}}},
\end{aligned}
$$

where

$$
\frac{1}{p_{1}}=\frac{1}{p_{0}}-\frac{1}{5}=1-\frac{4}{5 k}-\frac{1}{5}=\frac{4(k-1)}{5 k} \text { and } \frac{1}{q_{1}}=\frac{1}{q_{0}}-\frac{1}{10}=\frac{1}{2}-\frac{2}{5 k}-\frac{1}{10}=\frac{4(k-1)}{10 k} .
$$

On the other hand, from Lemma 2.3,

$$
\left\|u^{k-1}\right\|_{L_{x}^{p_{1}} L_{T}^{q_{1}}} \lesssim\|u\|_{L_{x}^{5 k / 4} L_{T}^{5 k / 2}}^{k-1} \lesssim\left\|D_{x}^{\alpha_{k}} D_{t}^{\beta_{k}} u\right\|_{L_{x}^{p_{k}} L_{T}^{q_{k}}}^{k-1} .
$$

Sobolev's inequality and (3.31) then imply

$$
\begin{aligned}
\left\|D_{x}^{s}\left(u^{k+1}\right)\right\|_{L_{x}^{1} L_{T}^{2}} & \lesssim\left\|D_{x}^{\alpha_{k}} D_{t}^{\beta_{k}} u\right\|_{L_{x}^{p_{k}} L_{T}^{q_{k}}}^{k}\left\|D_{x}^{s} u\right\|_{L_{x}^{5} L_{T}^{10}} \\
& \lesssim T^{k \gamma_{k}}\left\|D_{t}^{\gamma_{k}} D_{x}^{\alpha_{k}} D_{t}^{\beta_{k}} u\right\|_{L_{x}^{p_{k}} L_{T}^{q_{k}}}^{k}\left\|D_{x}^{s} u\right\|_{L_{x}^{5} L_{T}^{10}}
\end{aligned}
$$

Therefore,

$$
\left\|D_{x}^{s} \Phi(u)\right\|_{L_{x}^{2}} \leq\left\|D_{x}^{s} u_{0}\right\|_{L_{x}^{2}}+T^{k \gamma_{k}}\|u\|_{s}^{k+1} .
$$

To estimate the remainder norms in (3.27) we will make use of Lemmas 2.1, 2.2, 2.5 and 2.3 to lead to

$$
\|\Phi(u)\|_{s} \leq c\left\|u_{0}\right\|_{H^{s}}+c T^{k \gamma_{k}}\|u\|_{s}^{k+1} .
$$

Choose $a=2 c\left\|u_{0}\right\|_{H^{s}}$ and $T>0$ such that

$$
c a^{k} T^{k \gamma_{k}}<\frac{1}{20}
$$

This implies that $\Phi: X_{T}^{a} \rightarrow X_{T}^{a}$ is well defined. To finish the proof we need to prove that $\Phi$ is also a contraction; but, the argument is analogue to the previous one. The rest of the proof follows in a standard way.

Remark 3.1. From the proof of Theorem 1.1 it follows that

$$
T \sim\left\|u_{0}\right\|_{H^{s}}^{-1 / \gamma_{k}}=\left\|u_{0}\right\|_{H^{s}}^{-3 /\left(s-s_{k}\right)} .
$$

This is in agreement with the case $k=4$, where $T \sim\left\|u_{0}\right\|_{H^{s}}^{-3 / s}$ (see [9]). 


\section{Global well-posedness in $H^{1}$}

In this section, we intend to show Theorem 1.4. We begin by recalling the classical result obtained by Nagy [26] (see also Weinstein [29]), regarding the best constant of the Gagliardo-Nirenberg inequality.

Theorem 4.1. Let $k>0$, then the Gagliardo-Nirenberg inequality

$$
\|u\|_{L^{k+2}(\mathbb{R})}^{k+2} \leq K_{\mathrm{opt}}^{k+2}\|\nabla u\|_{L^{2}(\mathbb{R})}^{\frac{k}{2}}\|u\|_{L^{2}(\mathbb{R})}^{2+\frac{k}{2}},
$$

holds, and the sharp constant $K_{\mathrm{opt}}>0$ is explicitly given by

$$
K_{\mathrm{opt}}^{k+2}=\frac{k+2}{2\|\psi\|_{L^{2}}^{k}},
$$

where $\psi$ is the unique non-negative, radially-symmetric, decreasing solution of the equation

$$
\frac{k}{4} \Delta \psi-\left(1+\frac{k}{4}\right) \psi+\psi^{k+1}=0
$$

Proof. See [26] and [29].

Before proceeding to our main result, we will establish a relation between the solution $\psi$ of (4.35) and the unique non-negative, radially-symmetric, decreasing solution, $Q$, of the equation

$$
\Delta Q-Q+Q^{k+1}=0 .
$$

Remark 4.2. Recall that for the critical generalized KdV equation, that is, equation (1.1) with $k=4, \mu=1$, we have global solutions if $\left\|u_{0}\right\|_{L^{2}}<\|Q\|_{L^{2}}$, and $u_{0} \in H^{s}(\mathbb{R})$, $s>6 / 13$, where $Q$ is the solution of (4.36) with $k=4$ (see [25], [8], [9], and [29]).

First, we note that if $\psi$ is a solution of (4.35) then $\lambda \psi(\omega x)$, where $\lambda=\left(\frac{4}{k+4}\right)^{1 / k}$ and $\omega=\left(\frac{k}{k+4}\right)^{1 / 2}$, is a solution of (4.36). Therefore, by uniqueness, we have

$$
Q(x)=\lambda \psi(\omega x) .
$$

A simple calculation shows that

$$
\|Q\|_{L^{2}}^{2}=\frac{\lambda^{2}}{\omega}\|\psi\|_{L^{2}}^{2} .
$$

Combining this last relation with (4.34) yields

$$
K_{\mathrm{opt}}^{k+2}=\frac{2(k+2)(k+4)^{\frac{k-4}{4}}}{(k)^{\frac{k}{4}}\|Q\|_{L^{2}}^{k}} .
$$

Moreover, by multiplying (4.36) by $Q$, integrating, and applying integration by parts, we obtain

$$
\int Q^{k+2} d x=\|Q\|_{L^{2}}^{2}+\left\|\partial_{x} Q\right\|_{L^{2}}^{2}
$$


On the other hand, by multiplying (4.36) by $x \partial_{x} Q$, integrating, and applying integration by parts, we obtain the Pohozhaev-type identity

$$
\frac{2}{k+2} \int Q^{k+2} d x=\|Q\|_{L^{2}}^{2}-\left\|\partial_{x} Q\right\|_{L^{2}}^{2} .
$$

Combining the last two relations, we obtain

$$
\frac{(k+4)}{2(k+2)} \int Q^{k+2} d x=\|Q\|_{L^{2}}^{2} \text { and }\|Q\|_{L^{2}}^{2}=\frac{k+4}{k}\left\|\partial_{x} Q\right\|_{L^{2}}^{2} .
$$

Now we are ready to prove the main global result of this section.

Proof of Theorem 1.4. We proceed as follows: write the $\dot{H}^{1}$-norm of $u(t)$ using the quantities $M(u(t))$ and $E(u(t))$. Then we use the sharp Gagliardo-Nirenberg inequality (4.33) to yield

$$
\begin{aligned}
\left\|\partial_{x} u(t)\right\|_{L^{2}}^{2} & =2 E\left(u_{0}\right)+\frac{2}{k+2}\|u(t)\|_{L^{k+2}}^{k+2} \\
& \leq 2 E\left(u_{0}\right)+\frac{2}{k+2} K_{\mathrm{opt}}^{k+2}\left\|u_{0}\right\|_{L^{2}}^{\frac{k+4}{2}}\left\|\partial_{x} u(t)\right\|_{L^{2}}^{\frac{k}{2}}
\end{aligned}
$$

Let $X(t)=\left\|\partial_{x} u(t)\right\|_{L^{2}}^{2}, A=2 E\left(u_{0}\right)$, and $B=\frac{2}{k+2} K_{\text {opt }}^{k+2}\left\|u_{0}\right\|_{L^{2}}^{\frac{k+4}{2}}$, then we can write (4.39) as

$$
X(t)-B X(t)^{k / 4} \leq A, \text { for } t \in(0, T),
$$

where $T$ is given by Theorem 1.1.

Now let $f(x)=x-B x^{k / 4}$, for $x \geq 0$. The function $f$ has a local maximum at $x_{0}=\left(\frac{4}{k B}\right)^{4 /(k-4)}$ with maximum value $f\left(x_{0}\right)=\frac{k-4}{k}\left(\frac{4}{k B}\right)^{4 /(k-4)}$. If we require that

$$
2 E\left(u_{0}\right)<f\left(x_{0}\right) \text { and } X(0)<x_{0},
$$

the continuity of $X(t)$ implies that $X(t)<x_{0}$ for any $t$ as long as the solution exists.

Using relations (4.38), we have

$$
E(Q)=\frac{k-4}{2(k+4)}\|Q\|_{L^{2}}^{2} .
$$

Therefore, a simple calculation shows that conditions (4.41) are exactly the inequalities (1.10) and (1.11). Moreover the inequality $X(t)<x_{0}$ reduces to (1.12). The proof of Theorem 1.4 is thus completed.

\section{Global well-posedness in $H^{s}, s<1: \mu=-1$ and $k$ even}

In this section, we prove Theorem 1.5. As we mentioned in the introduction, we follow the "almost conservation law" scheme introduce in [5]-[7]. 
5.1. Modified energy functional. To start with, we introduce a substitute notion of "energy" that could be defined for less regular functions and that has very low increment in time. Given $s<1$ and a parameter $N \gg 1$, define a multiplier operator $I_{N}: H^{s} \rightarrow H^{1}$ such that

$$
\widehat{I_{N} f(\xi)} \equiv m_{N}(\xi) \widehat{f}(\xi),
$$

where the multiplier $m_{N}(\xi)$ is a non-decreasing in $|\xi|$, smooth and radially symmetric function defined as

$$
m_{N}(\xi)= \begin{cases}1 & , \text { if }|\xi| \leq N \\ \left(\frac{N}{|\xi|}\right)^{1-s} & , \text { if }|\xi| \geq 2 N\end{cases}
$$

To simplify the notation, we omit the dependence of $N$ in $I_{N}$ and denote it only by $I$. Note that the operator $I$ is smoothing of order $1-s$. Indeed we have

$$
\|u\|_{X_{s_{0}, b_{0}}} \leq c\|I u\|_{X_{s_{0}+1-s, b_{0}}} \leq c N^{1-s}\|u\|_{X_{s_{0}, b_{0}}},
$$

for any $s_{0}, b_{0} \in \mathbb{R}$.

Our substitute energy will be defined by $E^{1}(u)=E(I u)$. Obviously this energy makes sense even if $u$ is only in $H^{s}(\mathbb{R})$. Thus, in terms of $n$-linear functionals we have

$$
E^{1}(u)=-\frac{1}{2} \Lambda_{2}\left(m_{1} \xi_{1} m_{2} \xi_{2}\right)-\frac{\mu}{k+2} \Lambda_{k+2}\left(m_{1} \ldots m_{k+2}\right),
$$

where $m_{j}=m\left(\xi_{j}\right)$.

Thus, using the derivation law (2.26), we obtain

$$
\begin{aligned}
\frac{d}{d t} & E^{1}(u) \\
= & -\frac{1}{2}\left[\Lambda_{2}\left(m_{1} \xi_{1} m_{2} \xi_{2} \alpha_{2}\right)-2 \mu i \Lambda_{k+2}\left(m_{1} \xi_{1} m\left(\xi_{2}+\cdots+\xi_{k+2}\right)\left(\xi_{2}+\cdots+\xi_{k+2}\right)^{2}\right)\right] \\
& -\frac{\mu}{k+2}\left[\Lambda_{k+2}\left(m_{1} \ldots m_{k+2} \alpha_{k+2}\right)\right. \\
& \left.+\mu^{2} i \Lambda_{2 k+2}\left(m_{1} \ldots m_{k+1} m\left(\xi_{k+2}+\cdots+\xi_{2 k+2}\right)\left(\xi_{k+2}+\cdots+\xi_{2 k+2}\right)\right)\right] \\
= & -\frac{1}{2} \Lambda_{2}\left(m_{1} \xi_{1} m_{2} \xi_{2}\left(\xi_{1}^{3}+\xi_{2}^{3}\right)\right) \\
& +\frac{\mu i}{k+2} \Lambda_{k+2}\left(\left(m_{1}^{2} \xi_{1}^{3}+\cdots+m_{k+2}^{2} \xi_{k+2}^{3}\right)-m_{1} \ldots m_{k+2}\left(\xi_{1}^{3}+\cdots+\xi_{k+2}^{3}\right)\right) \\
& +\mu^{2} i \Lambda_{2 k+2}\left(m_{1} \ldots m_{k+1} m\left(\xi_{k+2}+\cdots+\xi_{2 k+2}\right)\left(\xi_{k+2}+\cdots+\xi_{2 k+2}\right)\right),
\end{aligned}
$$

where we have used the identity $\xi_{1}+\cdots+\xi_{k+2}=0$ and symmetrizing.

Remark 5.1. Observe that if $m=1$, the $\Lambda_{k+2}$ term vanish trivially. On the other hand, the terms $\Lambda_{2}$ and $\Lambda_{2 k+2}$ are also zero, since we have the restriction $\xi_{1}+\xi_{2}=0$ in the first and symmetrization in the later. This reproduces the Fourier proof of the energy conservation (1.9).

As one particular instance of the above computations and the Fundamental Theorem of Calculus, we have

$$
E^{1}(u)(t)-E^{1}(u)(0)=\int_{0}^{t} \frac{d}{d t} E^{1}(u)\left(t^{\prime}\right) d t^{\prime}=
$$




$$
\begin{aligned}
= & \frac{\mu i}{k+2} \int_{0}^{t} \Lambda_{k+2}\left(\left(m_{1}^{2} \xi_{1}^{3}+\cdots+m_{k+2}^{2} \xi_{k+2}^{3}\right)-m_{1} \ldots m_{k+2}\left(\xi_{1}^{3}+\ldots+\xi_{k+2}^{3}\right)\right)\left(t^{\prime}\right) d t^{\prime} \\
& +\mu^{2} i \int_{0}^{t} \Lambda_{2 k+2}\left(m_{1} \ldots m_{k+1} m\left(\xi_{k+2}+\cdots+\xi_{2 k+2}\right)\left(\xi_{k+2}+\cdots+\xi_{2 k+2}\right)\right)\left(t^{\prime}\right) d t^{\prime} .
\end{aligned}
$$

Most of our arguments here consist in showing that the quantity $E^{1}(u)$ is almost conserved in time.

Remark 5.2. We can think about $E^{1}(u)$ as the first generation of a family of modified energies. One can also define the "second energy"

$$
E^{2}(u)=-\frac{1}{2} \Lambda_{2}\left(m_{1} \xi_{1} m_{2} \xi_{2}\right)-\frac{\mu}{k+2} \Lambda_{k+2}\left(M_{k+2}\left(\xi_{1}, \ldots, \xi_{k+2}\right)\right),
$$

where $M_{k+2}$ is an arbitrary symmetric $(k+2)$-multiplier.

Thus, applying the derivation law (2.26), and choosing

$$
M_{k+2}\left(\xi_{1}, \ldots, \xi_{k+2}\right)=\frac{m_{1}^{2} \xi_{1}^{3}+\cdots+m_{k+2}^{2} \xi_{k+2}^{3}}{\xi_{1}^{3}+\cdots+\xi_{k+2}^{3}}
$$

we can force the term $\Lambda_{k+2}$, that shows up in the expression of $\frac{d}{d t} E^{2}(u)$, to be zero. Unfortunately the multiplier $M_{k+2}$ is not well defined in the set $\Gamma_{k+2}$. In fact, given $N \gg 1$, we can find numbers $\xi_{1}, \ldots, \xi_{k+2}$ such that the denominator of $M_{k+2}$ is zero and the numerator is different from zero (see [8, Proposition 3.1 and Remark 3.2]). Therefore, the refined approach introduced in [6] cannot be used in our setting.

5.2. Almost conservation law. This subsection presents a detailed analysis of the expression (5.44). The analysis identifies some cancelations in the pointwise upper bound of some multipliers depending on the relative size of the frequencies involved. Our aim is to prove the following almost conservation property.

Proposition 5.3. Let $s>1 / 2, N \gg 1$ and $u \in H^{s}(\mathbb{R})$ be a solution of (1.1) on $[T, T+\delta]$ so that $I u \in H^{1}(\mathbb{R})$. Then the following estimate holds

$$
\left|E^{1}(u)(T+\delta)-E^{1}(u)(T)\right| \lesssim N^{-2+}\left(\|I u\|_{X_{1, \frac{1}{2}+}^{\delta}}^{k+2}+\|I u\|_{X_{1, \frac{1}{2}+}^{\delta}}^{2 k+2}\right) .
$$

Remark 5.4. The exponent $-2+$ on the right hand side of (5.46) is directly tied to the restriction $s>\frac{3+2(1 / 2-2 / k)}{5}$ in our main theorem. If one could replace the increment $N^{-2+}$ by $N^{-\alpha+}$ for some $\alpha>0$ the argument we give in Section 5.3 implies global well-posedness of (1.1) for all $s>\frac{3+\alpha(1 / 2-2 / k)}{3+\alpha}$.

Proof. We start with the estimate for the $\Lambda_{k+2}$ term. Instead of estimating each multilinear expression separately, we shall exploit some cancelation between the two multipliers. Using symmetrization and the fact that $\xi_{1}+\cdots+\xi_{k+2}=0$ this term can be rewritten as

$$
\begin{aligned}
& \Lambda_{k+2}\left(\left(m_{1}^{2} \xi_{1}^{3}+\cdots+m_{k+2}^{2} \xi_{k+2}^{3}\right)-m_{1} \ldots m_{k+2}\left(\xi_{1}^{3}+\cdots+\xi_{k+2}^{3}\right)\right) \\
& =(k+2) \int_{\Gamma_{n}}\left(\frac{m\left(\xi_{2}+\cdots+\xi_{k+2}\right)}{m\left(\xi_{2}\right) \cdots m\left(\xi_{k+2}\right)}-1\right) \xi_{1}^{3} \widehat{\operatorname{Iu(\xi _{1})} \cdots I \widehat{u\left(\xi_{k+2}\right)}} .
\end{aligned}
$$


Therefore, our aim is to obtain the following inequality

$$
\operatorname{Term} \lesssim N^{-2+} \prod_{i=1}^{k+2}\left\|I \phi_{i}\right\|_{X_{1, \frac{1}{2}+}^{\delta}}
$$

where

$$
\text { Term } \left.\left.\equiv \mid \int_{0}^{\delta} \int_{\Gamma_{n}}\left(\frac{m\left(\xi_{2}+\cdots+\xi_{6}\right)}{m\left(\xi_{2}\right) \cdots m\left(\xi_{6}\right)}-1\right) \xi_{1}^{3} \widetilde{I \phi_{1}\left(\xi_{1}, t^{\prime}\right.}\right) \cdots I \widehat{\phi_{k+2}\left(\xi_{k+2}\right.}, t^{\prime}\right) d t^{\prime} \mid .
$$

We estimate Term as follows. Without loss of generality, we assume that the Fourier transforms of all these functions are non-negative. First, we bound the symbol in the parentheses pointwise in absolute value, according to the relative sizes of the frequencies involved. After that, the remaining integrals are estimated using Plancherel's formula, Hölder's inequality and Lemma 2.11. To sum over the dyadic pieces at the end we need to have extra factors $N_{j}^{0-}, j=1, \ldots, k+2$, everywhere.

We decompose the frequencies $\xi_{j}, j=1, \ldots, k+2$, into dyadic blocks $N_{j}$. By the symmetry of the multiplier

$$
\frac{m\left(\xi_{2}+\cdots+\xi_{k+2}\right)}{m\left(\xi_{2}\right) \cdots m\left(\xi_{k+2}\right)}-1
$$

in $\xi_{2}, \ldots, \xi_{k+2}$, we may assume that

$$
N_{2} \geq \cdots \geq N_{k+2}
$$

Moreover, we can assume $N_{2} \gtrsim N$, because otherwise the symbol is zero. The condition $\sum_{i=1}^{k+2} \xi_{i}=0$ implies $N_{1} \lesssim N_{2}$. We split the different frequency interaction into several cases, according to the size of the parameter $N$ in comparison to the $N_{i}$ 's.

Case $A: N_{2} \gtrsim N \gg N_{3} \geq \cdots \geq N_{k+2}$.

The condition $\sum_{i=1}^{k+2} \xi_{i}=0$ implies $N_{1} \sim N_{2}$. By the mean value theorem,

$$
\left|\frac{m\left(\xi_{2}\right)-m\left(\xi_{2}+\cdots+\xi_{k+2}\right)}{m\left(\xi_{2}\right)}\right| \lesssim \frac{\left|\nabla m\left(\xi_{2}\right)\left(\xi_{3}+\cdots+\xi_{k+2}\right)\right|}{m\left(\xi_{2}\right)} \lesssim \frac{N_{3}}{N_{2}} .
$$

Therefore, Lemma 2.11 and the Sobolev embedding imply that

$$
\begin{aligned}
\text { Term } & \lesssim \frac{N_{1}^{3} N_{3}}{N_{2}}\left\|I \phi_{1} I \phi_{3}\right\|_{L^{2}(\mathbb{R} \times[0, \delta])}\left\|I \phi_{2} I \phi_{4}\right\|_{L^{2}(\mathbb{R} \times[0, \delta])} \prod_{i=5}^{k+2}\left\|I \phi_{i}\right\|_{L^{\infty}} \\
& \lesssim \frac{N_{1}^{3} N_{3}}{N_{2} N_{1}^{2} N_{2}^{2}\left\langle N_{3}\right\rangle\left\langle N_{4}\right\rangle \prod_{i=5}^{k+2}\left\langle N_{i}\right\rangle^{1 / 2-}} \prod_{i=1}^{k+2}\left\|I \phi_{i}\right\|_{X_{1, \frac{1}{2}+}^{\delta}} \\
& \lesssim N^{-2+} N_{\max }^{0-} \prod_{i=1}^{k+2}\left\|I \phi_{i}\right\|_{X_{1, \frac{1}{2}+}^{\delta}},
\end{aligned}
$$

where $N_{\max }=\max \left\{N_{1}, \cdots, N_{k+2}\right\}$.

The remaining cases $N_{2} \gg N_{3} \gtrsim N$ and $N_{3} \geq \cdots \geq N_{6}($ Case $B)$ and $N_{2} \sim N_{3} \gtrsim$ $N$ and $N_{3} \geq \cdots \geq N_{6}$ (Case $C$ ) can be done using the same arguments as in Farah [8] (just put the remaining terms $I \phi_{j}, j=7, \cdots, k+2$ in $L_{x, t}^{\infty}$ and apply the Sobolev embedding). 
Now we turn to the estimate of the $\Lambda_{2 k+2}$ term. Before we start let us fix some notation. We write $N_{1}^{*} \geq N_{2}^{*} \geq N_{3}^{*}$ for the highest, second highest and third highest values of the frequencies $N_{1}, \ldots, N_{2 k+2}$. It is clear that

$$
\left|m_{1} \ldots m_{k+1} m\left(\xi_{k+2}+\cdots+\xi_{2 k+2}\right)\left(\xi_{k+2}+\cdots+\xi_{2 k+2}\right)\right| \lesssim N_{1}^{*}
$$

Again we perform a Littlewood-Paley decomposition of the functions $u$.

Case $A: N_{1}^{*} \sim N_{2}^{*} \sim N_{3}^{*} \gtrsim N$.

In view of (5.48) and the fact that $m^{3}\left(N_{1}^{*}\right) N_{1}^{* 3-} \gtrsim N^{3-}$, we have

$$
\begin{aligned}
\mid \int_{T}^{T+\delta} \Lambda_{2 k+2}\left(m_{1} \ldots\right. & \left.m_{k+1} m\left(\xi_{k+2}+\cdots+\xi_{2 k+2}\right)\left(\xi_{k+2}+\cdots+\xi_{2 k+2}\right)\right)\left(t^{\prime}\right) d t^{\prime} \mid \\
& \lesssim \frac{N_{1}^{* 0-}}{N^{2-}} \iint|J I u|^{3}|u|^{2 k-1} \\
& \lesssim \frac{N_{1}^{* 0-}}{N^{2-}}\|J I u\|_{L^{8}}^{3}\|u\|_{L^{8(2 k-1) / 5}}^{2 k-1} \\
& \lesssim \frac{N_{1}^{* 0-}\|I u\|_{X_{1, \frac{1}{2}+}^{3}}^{3}\|u\|_{X_{\alpha(8(2 k-1) / 5), \frac{1}{2}+}^{\delta(2-1}}^{2 k}}{N^{2-}}
\end{aligned}
$$

where we have applied Hölder inequality, (2.19) and (2.18).

Note that $\alpha(8(2 k-1) / 5)=(k-3) /(2 k-1)+$. Therefore the inequality $(5.42)$ implies

$$
\|u\|_{X_{\alpha(8(2 k-1) / 5), \frac{1}{2}+}^{\delta}} \lesssim\|I u\|_{X_{1, \frac{1}{2}+}^{\delta}}
$$

for all $s>(k-3) /(2 k-1)($ since $\alpha(8(2 k-1) / 5)+1-s \leq 1)$.

So, in this case

$$
\begin{gathered}
\left|\int_{T}^{T+\delta} \Lambda_{2 k+2}\left(m_{1} \ldots m_{k+1} m\left(\xi_{k+2}+\cdots+\xi_{2 k+2}\right)\left(\xi_{k+2}+\cdots+\xi_{2 k+2}\right)\right)\left(t^{\prime}\right) d t^{\prime}\right| \\
\lesssim \frac{N_{1}^{* 0-}}{N^{2-}}\|I u\|_{X_{1, \frac{1}{2}+}^{\delta+2}}^{2 k+2} .
\end{gathered}
$$

Case $B: N_{1}^{*} \sim N_{2}^{*} \gg N_{3}^{*}$.

Let $u_{j} \equiv u\left(N_{j}\right)$. Again, the inequality $m^{2}\left(N_{1}^{*}\right) N_{1}^{* 2-} \gtrsim N^{2-}$ and (5.48) implies that

$$
\begin{aligned}
\mid \int_{T}^{T+\delta} \Lambda_{2 k+2}\left(m_{1}\right. & \left.\ldots m_{k+1} m\left(\xi_{k+2}+\cdots+\xi_{2 k+2}\right)\left(\xi_{k+2}+\cdots+\xi_{2 k+2}\right)\right)\left(t^{\prime}\right) d t^{\prime} \mid \\
& \lesssim \frac{N_{1}^{* 0-}}{N^{1-}}\left\|J I u_{1} u_{3}\right\|_{L^{2}}\left\|J I u_{2} \prod_{j=4}^{2 k+2} u_{j}\right\|_{L^{2}} \\
& \lesssim \frac{N_{1}^{* 0-}}{N^{2-}}\left\|J I u_{1}\right\|_{L^{2}}\left\|u_{3}\right\|_{L^{2}}\left\|J I u_{2}\right\|_{L^{8}}\|u\|_{L^{8(2 k-1) / 3}}^{2 k-1} \\
& \lesssim \frac{N_{1}^{* 0-}}{N^{2-}}\|I u\|_{X_{1, \frac{1}{2}+}^{\delta}}^{2 k+2}
\end{aligned}
$$


where we have applied Hölder inequality, Lemma 2.11, (2.19) and (2.18) with $\alpha(8(2 k-$ $1) / 3)=(k-2) /(2 k-1)+<1 / 2$. This concludes the proof of Proposition 5.3.

5.3. Proof of Theorem 1.5. Before proceeding to the proof of Theorem 1.5 we will first establish local well-posedness for the generalized KdV equation (1.1) in the Bourgain spaces $X_{s, b}$. As in Theorem 1.1, by the Duhamel's principle, we need to find a solution for the following integral equation

$$
u(t)=U(t) u_{0}+\int_{0}^{t} U\left(t-t^{\prime}\right) \partial_{x}\left(u^{k+1}\right)\left(t^{\prime}\right) d t^{\prime} .
$$

To work in the $X_{s, b}$ spaces we consider another version of (5.49), that is,

$$
u(t)=\varphi(t) U(t) u_{0}+\varphi_{T}(t) \int_{0}^{t} U\left(t-t^{\prime}\right) \partial_{x}\left(u^{k+1}\right)\left(t^{\prime}\right) d t^{\prime} .
$$

Note that the integral equation (5.50) is defined for all $(x, t) \in \mathbb{R}^{2}$. Moreover if $u$ is a solution of $(5.50)$ than $\tilde{u}=\left.u\right|_{[0, T]}$ will be a solution of $(5.49)$ in $[0, T]$.

The proof proceeds by the usual fixed point argument. Applying Lemma 2.10, we have for all $s>s_{k}$,

$$
\begin{aligned}
\|u\|_{X_{s, 1 / 2+}} & =\left\|\varphi(t) U(t) u_{0}+\varphi_{T}(t) \int_{0}^{t} U\left(t-t^{\prime}\right) \partial_{x}\left(u^{k+1}\right)\left(t^{\prime}\right) d t^{\prime}\right\|_{X_{s, 1 / 2+}} \\
& \lesssim\left\|u_{0}\right\|_{H^{s}}+T^{\varepsilon}\left\|\partial_{x}\left(u^{k+1}\right)\right\|_{X_{s,-1 / 2++}},
\end{aligned}
$$

for sufficiently small $\varepsilon>0$.

Thus, the crucial nonlinear estimate for the local existence is given in the following lemma.

Lemma 5.5. For $s>s_{k}=(k-4) / 2 k$, we have

$$
\left\|\partial_{x}\left(u^{k+1}\right)\right\|_{X_{s,-\frac{1}{2}++}} \lesssim\|u\|_{X_{s, \frac{1}{2}+}^{k+1}}^{k} .
$$

Proof. By the fractional Leibniz rule in Lemma 2.6, inequality (2.17), and Hölder's inequality, we obtain

$$
\begin{aligned}
\left\|\partial_{x}\left(u^{k+1}\right)\right\|_{X_{s,-\frac{1}{2}++}} & =\left\|J^{s} \partial_{x}\left(u^{k+1}\right)\right\|_{X_{0,-\frac{1}{2}++}} \lesssim\left\|J^{s} \partial_{x}\left(u^{k+1}\right)\right\|_{L_{x}^{5 / 4+} L_{t}^{10 / 9+}} \\
& \lesssim\left\|J^{s}\left(u^{k}\right)\right\|_{L_{x}^{p_{1}+} L_{t}^{q_{1}+}}\left\|\partial_{x} u\right\|_{L_{x}^{p_{2}} L_{t}^{q_{2}}}+\left\|u^{k}\right\|_{L_{x}^{5 / 4+} L_{t}^{5 / 2+}}\left\|J^{s} \partial_{x} u\right\|_{L_{x}^{\infty} L_{t}^{2}} \\
& \lesssim\left\|u^{k-1}\right\|_{L_{x}^{5 k / 4(k-1)+} L_{t}^{5 k / 2(k-1)++}}\left\|J^{s} u\right\|_{L_{x}^{p_{3}} L_{t}^{q_{3}}}\left\|\partial_{x} u\right\|_{L_{x}^{p_{2} L_{t}^{q_{2}}}} \\
& +\|u\|_{L_{x}^{5 k / 4} L_{t}^{5 k / 2}}^{k-1}\|u\|_{L_{x}^{5 k / 4+} L_{t}^{5 k / 2+}}\left\|J^{s} \partial_{x} u\right\|_{L_{x}^{\infty} L_{t}^{2}}
\end{aligned}
$$

where $p_{2}$ and $p_{3}$ are defined as in (2.24).

Therefore, an application of inequalities (2.22) and (2.23) followed by inequalities (2.20) and (2.21) yield the desired estimate (5.51).

Remark 5.6. As a consequence, one can recover all the well known range of existence for the local theory in terms of the $X_{s, b}$ spaces. 
Next, we consider the following modified equation

$$
\left\{\begin{array}{l}
I u_{t}+I u_{x x x}-I\left(u^{k+1}\right)_{x}=0, \quad x \in \mathbb{R}, t>0 \\
I u(x, 0)=I u_{0}(x)
\end{array}\right.
$$

Clearly if $I u \in H^{1}(\mathbb{R})$ is a solution of $(5.52)$, then $u \in H^{s}(\mathbb{R})$ is a solution of (1.1) in the same time interval. Therefore, we need to prove that, in fact, the above modified equation has a global solution.

Applying the interpolation lemma (see [7, Lemma 12.1]) to (5.51), we obtain

$$
\left\|\partial_{x} I\left(u^{k+1}\right)\right\|_{X_{1,-1 / 2++}} \lesssim\|I u\|_{X_{1,1 / 2+}+1}^{k+1} .
$$

where the implicit constant is independent of $N$. Now, standard arguments invoking the contraction-mapping principle give the following variant local well-posedness result.

Theorem 5.7. Assume $s_{k}<s<1$. Let $u_{0} \in H^{s}(\mathbb{R})$ be given. Then there exists a positive number $\delta$ such that the IVP (5.52) has a unique local solution Iu $\in C([0, \delta]$ : $\left.H^{1}(\mathbb{R})\right)$ such that

$$
\|I u\|_{X_{1, \frac{1}{2}+}^{\delta}} \lesssim\left\|I u_{0}\right\|_{H^{1}} .
$$

Moreover, the existence time can be estimated by

$$
\delta \sim \frac{1}{\left\|I u_{0}\right\|_{H^{1}}^{\sigma}}
$$

where $\sigma>0$.

Now, we have all tools to prove our global result stated in Theorem 1.5.

Proof of Theorem 1.5. Let $u_{0} \in H^{s}(\mathbb{R})$ with $s_{k}<s<1$. Our goal is to construct a solution to (5.52) (and therefore to (1.1)) on an arbitrary time interval $[0, T]$. We rescale the solution by writing $u_{\lambda}(x, t)=\lambda^{-2 / k} u\left(x / \lambda, t / \lambda^{3}\right)$. We can easily check that $u(x, t)$ is a solution of (1.1) on the time interval $[0, T]$ if and only if $u_{\lambda}(x, t)$ is a solution to the same equation, with initial data $u_{0, \lambda}=\lambda^{-2 / k} u_{0}(x / \lambda)$, on the time interval $\left[0, \lambda^{3} T\right]$.

Since $k$ is even we have $\int u^{k+2}(x, t) d x>0$, for all $t>0$, therefore for $\mu=-1$

$$
\left\|\partial_{x} I u_{\lambda}(t)\right\|_{L^{2}}^{2} \lesssim E\left(I u_{\lambda}\right)(t) .
$$

On the other hand

$$
\begin{aligned}
E\left(I u_{0, \lambda}\right) & \lesssim\left\|\partial_{x} I u_{0, \lambda}\right\|_{L^{2}}^{2}+\left\|I u_{0, \lambda}\right\|_{L^{k+2}}^{k+2} \\
& \lesssim\left(N^{2(1-s)} \lambda^{-2(s-1 / 2+2 / k)}+\lambda^{-(k+2)(2 / k-1 /(k+2))}\right)\left(1+\left\|u_{0}\right\|_{H^{s}}\right)^{k+2} .
\end{aligned}
$$

where in the last inequality we have used that

$$
\left\|\partial_{x} I u_{0, \lambda}\right\|_{L^{2}} \lesssim N^{1-s}\left\||\xi|^{s} \widehat{u_{0, \lambda}}\right\|_{L^{2}}=N^{1-s} \lambda^{-(s-1 / 2+2 / k)}\left\|u_{0}\right\|_{\dot{H}^{s}} .
$$

and, by Sobolev's embedding,

$$
\left\|I u_{0, \lambda}\right\|_{L^{k+2}} \lesssim\left\|D_{x}^{1 / 2-1 / k+2} I u_{0, \lambda}\right\|_{L^{2}} \lesssim \lambda^{-(2 / k-1 /(k+2))}\left\|u_{0}\right\|_{H^{s}}
$$

for all $s>1 / 2-1 / k+2$. 
Now, we apply our variant local existence Theorem 5.7 on $[0, \delta]$, where $\delta \sim$ $\left\|I u_{0, \lambda}\right\|_{H^{1}}^{-\sigma}, \sigma>0$, to conclude that

$$
\left\|I u_{\lambda}\right\|_{X_{1, \frac{1}{2}+}^{\delta}} \lesssim\left\|I u_{0, \lambda}\right\|_{H^{1}}
$$

The choice of the parameter $N=N(T)$ will be made later, but we select $\lambda$ now by requiring

$$
N^{2(1-s)} \lambda^{-2(s-1 / 2+2 / k)}\left(1+\left\|u_{0}\right\|_{H^{s}}\right)^{k+2}<1 \Longrightarrow \lambda \sim N^{\frac{1-s}{s-1 / 2+2 / k}} .
$$

In this case $E\left(I u_{0, \lambda}\right) \leq 1$ and $\left\|I u_{0, \lambda}\right\|_{H^{1}} \leq 1$.

Remark 5.8. Note that $2 / k-1 /(k+2)>0$.

From now on, we drop the $\lambda$ subscript on $u$. By the almost conservation law stated in Proposition 5.3 and (5.55)-(5.57), we have

$$
E^{1}(1) \leq E^{1}(0)+c N^{-2+}<1+c N^{-2+}<2 .
$$

We iterate this process $M$ times obtaining

$$
E^{1}(M) \leq E^{1}(0)+c M N^{-2+}<1+c M N^{-2+}<2,
$$

as long as $M N^{-2+} \lesssim 1$, which implies that the lifetime of the local results remains uniformly of size 1 . We take $M \sim N^{2-}$. This process extends the local solution to the time interval $\left[0, N^{2-}\right]$. Now, we choose $N=N(T)$ so that

$$
N^{2-}>\lambda^{3} T \sim N^{3\left(\frac{1-s}{s-1 / 2+2 / k}\right)} T \Longrightarrow N^{2-3 \frac{1-s}{s-1 / 2+2 / k}-}>T .
$$

Therefore, if $s>\frac{4(k-1)}{5 k}$ then $T$ can be taken arbitrarily large which conclude our global result.

Finally, we need to establish the polynomial bound (1.13). Undoing the scaling, we have

$$
\left\|\partial_{x} I u_{\lambda}\left(\lambda^{3} T_{0}\right)\right\|_{L^{2}}^{2}=\frac{1}{\lambda^{1+4 / k}}\left\|\partial_{x} I u\left(T_{0}\right)\right\|_{L^{2}}^{2} .
$$

Let $T_{0} \sim N^{2-3 \frac{1-s}{s-1 / 2+2 / k}-}$, therefore our uniform bound (5.58) together with (5.42), (1.8), and (5.54) imply

$$
\begin{aligned}
\left\|u\left(T_{0}\right)\right\|_{H^{s}}^{2} \lesssim\left\|I u\left(T_{0}\right)\right\|_{H^{1}}^{2} & \lesssim\left\|I u\left(T_{0}\right)\right\|_{L^{2}}^{2}+\left\|\partial_{x} I u\left(T_{0}\right)\right\|_{L^{2}}^{2} \\
& \lesssim\left\|u_{0}\right\|_{L^{2}}^{2}+\lambda^{1+4 / k}\left\|\partial_{x} I u_{\lambda}\left(\lambda^{3} T_{0}\right)\right\|_{L^{2}}^{2} \\
& \lesssim\left\|u_{0}\right\|_{L^{2}}^{2}+N^{(1+4 / k)}\left(\frac{1-s}{s-1 / 2+2 / k}\right) \\
& \lesssim\left(1+T_{0}\right)^{\frac{(1+4 / k)(1-s)}{5 s-4(k-1) / k}+}\left(1+\left\|u_{0}\right\|_{H^{s}}\right)^{2} .
\end{aligned}
$$

The proof of Theorem 1.5 is thus completed.

Remark 5.9. It is not clear how to apply the I-method when $\mu=1$ or $\mu=-1$ and $k$ odd. In these cases, we may not have inequality (5.54). Therefore, to perform the interactions explained above we need to verify the hypotheses of Theorem 1.4 for the modified solution Iu(t) at each step. However, the only available estimate in the homogeneous $H^{s}$-Sobolev space is the following

$$
\left\|\partial_{x} I u_{t}\right\|_{L^{2}} \lesssim N^{1-s}\|u(t)\|_{\dot{H}^{s}} .
$$


Since, at the end of the argument we need to take $N$ large, inequalities (1.10)-(1.12) are not satisfied during all the interactions.

\section{Acknowledgements}

The authors thank the referee for helpful comments and suggestions which improved the presentation of the paper.

\section{References}

[1] B. Birnir, C. E. Kenig, G. Ponce, N. Svanstedt, and L. Vega, On the ill-posedness of the IVP for the generalized Korteweg-de Vries and nonlinear Schrödinger equations, J. London Math. Soc. 53, 551-559, 1996.

[2] J. Bourgain, Fourier transform restriction phenomena for certain lattice subsets and applications to nonlinear evolution equations, I and II, Geom. Funct. Anal. 3, 107-156, 209-262, 1993.

[3] J. Bourgain, Refinements of Strichartz' inequality and applications to 2D-NLS with critical nonlinearity, Internat. Math. Res. Notices 1998, 253-283, 1998.

[4] M. Christ, J. Colliander, and T. Tao, Asymptotics, frequency modulation and low regularity ill-posedness for canonical defocusing equations, Amer. J. Math. 125, 1235-1293, 2003.

[5] J. Colliander, M. Keel, G. Staffilani, H. Takaoka, and T. Tao, Almost conservation laws and global rough solutions to a nonlinear Schrödinger equation, Math. Res. Lett. 9, 659-682, 2002.

[6] J. Colliander, M. Keel, G. Staffilani, H. Takaoka, and T. Tao, Sharp global well-posedness for $\mathrm{KdV}$ and modified KdV on $\mathbb{R}$ and $\mathbb{T}$, J. Amer. Math. Soc. 16, 705-749, 2003.

[7] J. Colliander, M. Keel, G. Staffilani, H. Takaoka, and T. Tao, Multilinear estimates for periodic KdV equations, and applications, J. Funct. Anal. 211, 173-218, 2004.

[8] L. G. Farah, Global rough solutions to the critical generalized kdv equation, J. Differential Equations 249, 1968-1985, 2010.

[9] G. Fonseca, F. Linares, and G. Ponce, Global existence for the critical generalized KdV equation, Proc. Amer. Math. Soc. 131, 1847-1855, 2003.

[10] A. Grünrock, M. Panthee, and J. D. Silva, A remark on global well-posedness below $L^{2}$ for the GKDV-3 equation, Differential Integral Equations 20, 1229-1236, 2007.

[11] A. Grünrock, A bilinear Airy-estimate with application to gKdV-3, Differential Integral Equations 18, 1333-1339, 2005.

[12] Z. Guo, Global well-posedness of Kortewegde Vries equation in $H^{-3 / 4}(\mathbb{R})$, J. Math. Pures Appl. 91, 583-597, 2009

[13] J. Holmer and S. Roudenko, A sharp condition for scattering of the radial 3D cubic nonlinear Schrödinger equation, Commun. Math. Phys. 282, 435-467, 2008.

[14] T. Kato, On the Cauchy problem for the (generalized) Korteweg-de Vries equation, Studies in applied mathematics, volume 8 of Adv. Math. Suppl. Stud., 93-128, Academic Press, New York, 1983.

[15] C. E. Kenig and F. Merle, Global well-posedness, scattering, and blow-up for the energy-critical focusing nonlinear Schrödinger equation in the radial case, Invent. Math. 166, 645-675, 2006.

[16] C. E. Kenig, G. Ponce, and L. Vega, Oscillatory integrals and regularity of dispersive equations, Indiana Univ. Math. J. 40, 33-69, 1991.

[17] C. E. Kenig, G. Ponce and L. Vega, Well-posedness and scattering results for the generalized Korteweg-de Vries equation via the contraction principle, Comm. Pure Appl. Math. 46, 527-620, 1993.

[18] C. E. Kenig, G. Ponce and L. Vega, A bilinear estimate with applications to the KdV equation, J. Amer. Math. Soc. 9, 573-603, 1996.

[19] C. E. Kenig, G. Ponce, and L. Vega, On the ill-posedness of some canonical dispersive equations, Duke Math. J. 106, 617-633, 2001.

[20] C. E. Kenig, G. Ponce, and L. Vega, On the concentration of blow up solutions for the generalized KdV equation critical in $L^{2}$, Contemp. Math. 263, 131-156, 2000.

[21] H. Koch and J. L. Marzuola, Small data scattering and soliton stability in $\dot{H}^{-\frac{1}{6}}$ for the quartic KdV equation, arXiv:1001.4747v2 [math.AP]. 
[22] D. Korteweg and G. de Vries, On the change of form of long waves advancing in a rectangular canal, and on a new type of long stationary waves, Philos. Mag. 539, 422-443, 1895.

[23] Y. Martel and F. Merle, Blow up in finite time and dynamics of blow up solutions for the $L^{2}$-critical generalized KdV equation, J. Amer. Math. Soc. 15, 617-664, 2002.

[24] F. Merle, Existence of blow-up solutions in the energy space for the critical generalized KdV equation, J. Amer. Math. Soc. 14, 555-578, 2001.

[25] C. Miao, S. Shao, Y. Wu, and G. Xu, The low regularity global solutions for the critical generalized KdV equation, arXiv:0908.0782v3 [math.AP].

[26] B. V. Sz. Nagy, Über Integralgleichungen zwischen einer Funktion und ihrer Ableitung, Acta Sci. Math. 10, 64-74, 1941.

[27] T. Tao, Scattering for the quartic generalised Korteweg-de Vries equation, J. Differential Equations 3, 623-651, 2006.

[28] N. Tzvetkov, Remark on the local ill-posedness for KdV equation, C. R. Acad. Sci. Paris 329, 1043-1047, 1999.

[29] M. Weinstein, Nonlinear Schrödinger equations and sharp interpolation estimates, Comm. Math. Phys. 87, 567-576, 1983.

ICEx, Universidade Federal de Minas Gerais, Av. Antônio Carlos, 6627, Caixa Postal 702, 30123-970, Belo Horizonte-MG, Brazil

E-mail address: lgfarah@gmail.com

impa, Estrada Dona Castorina 110, CeP 22460-320, Rio de Janeiro, RJ, Brazil.

E-mail address: linares@impa.br

IMECC-UNiCAMP, Rua SÉrgio Buarque de Holanda, 651, 13083-859, Campinas-SP, BraZIL

E-mail address: apastor@ime.unicamp.br 